\title{
Stay home and stay active? The impact of stay-at- home restrictions on physical activity routines in the UK during the COVID-19 pandemic
}

\section{Victoria Eshelby, Muhammed Sogut, Kate Jolly, Ivo Vlaev \& Mark T. Elliott}

To cite this article: Victoria Eshelby, Muhammed Sogut, Kate Jolly, Ivo Vlaev \& Mark T. Elliott (2021): Stay home and stay active? The impact of stay-at-home restrictions on physical activity routines in the UK during the COVID-19 pandemic, Journal of Sports Sciences, DOI: 10.1080/02640414.2021.1992885

To link to this article: https://doi.org/10.1080/02640414.2021.1992885
(c) 2021 The Author(s). Published by Informa UK Limited, trading as Taylor \& Francis Group.
曲 Published online: 31 Oct 2021.

Џll Article views: 187

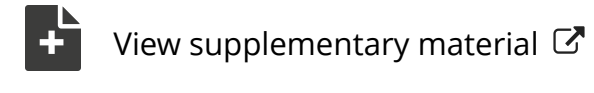

View supplementary material 다

Submit your article to this journal 


\title{
Stay home and stay active? The impact of stay-at-home restrictions on physical activity routines in the UK during the COVID-19 pandemic
}

\author{
Victoria Eshelby ${ }^{\mathrm{a}}$, Muhammed Sogut ${ }^{\mathrm{a}}$, Kate Jolly $\mathbb{D}^{\mathrm{b}}{ }^{\mathrm{b}}$, Ivo Vlaev $\mathbb{D}^{\mathrm{c}}$ and Mark T. Elliott $\mathbb{D}^{\mathrm{a}}$ \\ anstitute of Digital Healthcare, WMG, University of Warwick, Coventry, UK; ${ }^{b}$ Institute of Applied Health Research, University of Birmingham, \\ Birmingham, UK; 'Warwick Business School, University of Warwick, Coventry, UK
}

\begin{abstract}
We investigated which population groups were impacted most in terms of physical activity levels during the restrictions applied during the COVID-19 pandemic. We surveyed UK residents, sampled through users of a rewards-for-exercise app (Sweatcoin; $n=749$ ) and an online panel (Prolific; $n=907$ ). Of the app users, $n=487$ further provided daily step-count data collected by the app, prior to, and during the periods of restrictions in the UK between March-June 2020. Regression models were used to investigate factors associated with self-reported change in physical activity and change in daily step-count during the periods of restrictions. Significant factors associated with self-reported change in physical activity included rural residents (positive, $b=0.87, p<0.001$ ), relative to urban dwellers, people classed as obese (negative, $b=-0.51, p=0.008$, relative to healthy weight) and gym users (negative, $b=-1.10, p<0.001$, relative to walkers). All groups had reduced step counts during restrictions, with Black, Asian and minority ethnic groups showing greater reductions compared to White British ethnicity (negative, $b=-0.18, p$ $=0.008$ ). Targeted interventions are required to ensure that physical and mental health impacts of sedentary behaviour are not exacerbated over the long-term by significant reductions in physical activity identified in these groups particularly those who are also more vulnerable to COVID-19.
\end{abstract}

ARTICLE HISTORY

Accepted 8 October 2021

KEYWORDS

COVID-19; physical activity; step-count; restrictions; exercise habits

\section{Introduction}

Throughout the period of the COVID-19 pandemic, the UK government introduced restrictions as a means to slow the progression of the outbreak. The first phase of restrictions was applied from 23 March 2020 with a "Stay at Home" message. Travel was limited to all but essential journeys, and all non-essential services, including sports and leisure facilities were closed; outdoor exercise was permitted once per day (Prime Minister's statement on coronavirus (COVID-19), 2020a). A large proportion of the population switched to working from home (Coronavirus and homeworking in the UK - Office for National Statistics, 2020) while many others were furloughed (Comparison of furloughed jobs data - Office for National Statistics, 2020). On the next phase of lockdown restrictions (introduced May 13th, 2020), the government reopened outdoor public places, allowed people to exercise more than once a day and to drive to outdoor destinations. However, gyms and sports facilities remained closed (Prime Minister's statement on coronavirus (COVID-19), 2020b). The result of the COVID-19 pandemic has drastically disrupted routines in adults and children around the globe ranging from commuting behaviours to recreational habits (Maltagliati et al., 2020). This has had a subsequent impact on people's physical activity (PA) routines, due to government level restrictions put in place to stop the spread of the virus (Cheval et al., 2020; Di Corrado et al., 2020, p. 7,8; Dunton et al., 2020; Teran-Escobar et al., 2021).
Growing literature has identified a decrease in self-reported PA as a direct result of the lock-downs resulting from the COVID-19 pandemic (Caputo \& Reichert, 2020). A general trend has been observed that individuals have shifted from moderate physical exercise to a more sedentary lifestyle across all countries studied. Naturally, a decline in physical exercise is of great concern. The effects of PA in improving physical health is well documented and paired with severe COVID-19 symptoms being associated with obesity, places great importance on PA within the pandemic landscape (Jakobsson et al., 2020). Individuals who rely on gyms and sport facilities have been expected to deviate into alternative forms of PA, compliant with governmental restrictions. Consequently, this could explain the shift towards a sedentary lifestyle, as confirmed in many self-reported surveys as well as objectively measured PA, with the step-count in many populations dropping by as much as $15 \%$ in the first 30 days of the lockdown (Tison et al., 2020).

The differences in restrictions and governmental phases provides an interesting landscape to observe the routine changes between different social demographics. In this study we investigate the changes in PA routines people within the UK have experienced during the periods of lockdown due to the COVID-19 pandemic. The previous literature exploring PA during the pandemic often report on one facet of measurement e.g., self-reported surveys (Caputo \& Reichert, 2020; Cross et al., 2021). In contrast here, we compare both self-report and objective measures, in terms of step-count, to investigate how the 
restrictions have impacted on $\mathrm{PA}$ levels during the first half of 2020. We were particularly interested to determine which groups of people had increased PA during lockdown compared to those who had reduced levels of PA. We compared individuals' PA levels across two phases of restrictions: the main lockdown period (Phase 1), and the somewhat relaxed restrictions to outdoor exercise (Phase 2 ) relative to a period shortly prior to the Phase 1 lockdown. To do this we captured a broad range of grouping variables through the questionnaire, in addition to the self-reported and objective measures of PA. We briefly describe the variables along with justification for their inclusion below.

Demographics: Both age and ethnicity have been identified as important factors that affect vulnerability to COVID-19, with older adults having a substantially higher risk of hospitalisation and death than young adults due to COVID-19 (Docherty et al., 2020). Similarly, Black, Asian and Minority Ethnic (BAME) also had a higher risk of severe effects, compared to White ethnicity groups (Niedzwiedz, O'Donnell, Jani et al., 2020). Therefore, we wanted to investigate how these groups were also being impacted in terms of PA levels during the lockdowns. We also considered residential location to be an important factor and hypothesised that those in urban areas may show greater negative impact, due to potentially relying on the use of gyms and sports facilities in city centre locations, compared to those living rurally. Related to this, we further investigated the primary form of PA respondents participated in prior to (and during) lockdown to understand how the groups relying on facilities during the restrictions were impacted.

Mental and Physical Health: Supported by the strong evidence of the relationship between levels of PA and mental wellbeing, we predicted that increases in PA reported in lockdown would correlate with participants reporting greater mental wellbeing (Biddle et al., 2021). In addition, we captured physical traits including body mass index and whether participants had had COVID-19 to determine how this impacted and change in PA levels during the lockdown periods, particularly due to the potential for inactivity to exacerbate symptoms (Woods et al., 2020).

Working Status: Many people's work routines were impacted by the restrictions put in place during the pandemic. In the UK, those whose place of work was closed were put on furlough and remained at home; key workers continued to work at their usual place while many office-based workers begun working from home (Coronavirus and homeworking in the UK - Office for National Statistics, 2020). This change in routine is likely to have impacted directly on PA, particularly through changes in commuting patterns. On the one hand active commuting could have reduced due to more people working from home, but on the other, the increased anxiety of using public transport is likely to have increased use of active transport modes such as walking or cycling in those who continued to commute during the lockdown (Harrington \& Hadjiconstantinou, 2020).

Personality: In studies of the relationship between PA and personality traits, it has been suggested that higher Extraversion and lower Neuroticism are positively related to PA levels (Rhodes, 2006); similarly Extraversion and Consciousness have been suggested to be positively related with exercise intention-behaviour (Hoyt et al., 2009). Therefore, we predicted that individuals scoring highly on Extraversion and Consciousness may be more likely to adapt their PA behaviour regardless of restrictions and hence be more likely to have increased or maintained PA levels.

Collecting this broad set of variables has allowed us to use an integrative approach that identifies populations most impacted from the restrictions, such that future interventions can be developed to help them adapt and maintain or increase PA levels.

\section{Methods}

\section{Participants}

Participants were recruited from two sources. The first recruitment source was via a physical activity incentives app (Sweatcoin (Derlyatka et al., 2019); $n=1322$ ). This app rewards users according to their step-count recorded by the inbuilt functionality of the smartphone (i.e. Apple Healthkit on iOS devices or Google Fit on Android devices) and additionally validated by the app's bespoke algorithms (Derlyatka et al., 2019). Users were recruited using an advert placed on the inapp marketplace, where the rewards are offered. They were able to click the advert to receive the link to the participant information and subsequently consent and continue to complete the questionnaire. The participants were further given the option of providing their historic step count data recorded by the smartphone and logged by the app. The step count data covered the period between 1 February 2020 and the date of completing the survey. A total of 950 users consented to providing this data in addition to their survey responses. After matching data to survey responses and removing entries with more than $50 \%$ of days with missing step-count values, 487 participants were used for the additional analyses of objective data (in combination with their survey responses).

The second source of participants was through a survey panel (Prolific (Palan \& Schitter, 2018); $n=932$ ). Through this platform, the survey was available to any panel members who were adults that resided in the UK. After removal of duplicate and incomplete entries, a total of 1656 survey responses were used for analysis of self-reported measures (app: $n=749$, panel: $\mathrm{n}=907)$.

\section{Ethics}

The study was given ethical approval by the Humanities and Social Sciences Research Ethics Committee at the University of Warwick. Informed consent was given by participants before proceeding with the survey questions. Each participant was provided with a nominal payment of $£ 2$ for fully completing the survey, which took approx. 10-15 minutes. Participants provided additional consent for sharing step-count data, by ticking a box on the consent form.

\section{Periods of study}

We investigated the lockdown period between March and May 2020 relative to the period just before restrictions were put in place. As the government relaxed some restrictions just prior to 
the survey taking place, we captured retrospective results based on three periods (Table 1): Pre-restrictions (baseline period), Phase 1 (Full restrictions: gyms, facilities closed; one period of exercise a day), Phase 2 (Partial restrictions: gyms, facilities closed; unlimited outdoor exercise). We were primarily interested in how the restrictions impacted on people's usual PA routines and therefore captured change in PA in Phases 1 and 2, relative to the baseline period. We captured the Phase 2 as well as Phase 1 data to analyse whether the relaxation of some restrictions changed notably changed the impact on PA levels and whether this varied across different groups of individuals.

\section{Questionnaire design}

The questionnaire consisted of demographic, wellbeing, physical activity, working status, COVID-19 status and opinions, and personality information. Participants completed the questionnaire once during the period between May $29^{\text {th }}$ to 10 June 2020. They were asked to consider their responses retrospectively to three time periods that occurred prior to and during lockdown (see Table 1).

The following variables were collected in the survey data (A full sample breakdown is given in Supplementary Information A).

\section{General demographics}

We captured gender, age, height, weight and ethnicity information. In addition, we gathered participants' geographic location using the initial part of their postcode, along with details on whether they lived in an urban, suburban or rural location and whether they had access to a private garden. Finally, we captured whether they had children (under the age of 18) living at home.

\section{Wellbeing}

We used the four measures of personal wellbeing (Office of National Statistics, (Waldron, 2010)) to measure self-reported measures of Life Satisfaction, Worthwhile, Happiness and Anxiety on a scale of 0 (not at all) to 10 (completely). In addition, we asked users to rate their overall health on that day, on a scale of 0 (worst health) to 100 (best health).

\section{Working status}

We asked participants about their current work status over the past week, in terms of whether they were working from home, working at their usual location (away from home), furloughed, a student or not in employment.

\section{COVID-19 status and opinions}

We asked participants how worried they were about coronavirus and to rate their certainty on whether they had or previously had COVID-19 (or not). Participants further stated whether they had a received a letter stating that they should follow shielding guidelines, and whether they were complying with this. Similarly, we captured the proportion of people participants thought were complying with social distancing measures and government-imposed restrictions of movements.

\section{Personality}

The Big-Five personality dimensions (openness to experience, conscientiousness, extraversion, agreeableness, neuroticism) (De Raad, 2000) were captured using the Ten Item Personality Measure (TIPI; (Gosling et al., 2003)). In addition we investigated their attitude to long versus short-term rewards, where participants stated a preference to receiving one month's wages immediately or two-month's wages in 12-month's time.

\section{Physical activity}

Participants were asked the following regarding their PA routine:

\section{Types of exercise}

We asked participants to define the main form of exercise they routinely participated in over the three time periods.

\section{Time spent on activities}

Number of hours spent weekly on physical exercise (e.g., swimming, jogging, football, aerobics, gym), cycling and walking.

\section{Likelihood to stick with new routine}

Participants rated how likely they were to return to their original physical activity routine (prior to restrictions) or their new routine (during the second phase of restrictions) once all restrictions were lifted and business had reopened.

\section{Commuting related physical activity}

Participants were asked to state the number of minutes spent walking, cycling, using public transport and driving, during their commute to work. Participants who spent more than 5 minutes either walking or cycling during their journey to work were classified as active commuters.

\section{Self-reported change in physical activity}

Finally, participants were asked to consider their PA based on the three periods relating to times prior to lockdown and two periods of UK government restrictions between March and June

Table 1. Based on the level of restrictions, three periods were analysed. The date range is based on the start/end date of restrictions coming into effect, based on UK Government announcements (Prime Minister's statement on coronavirus (COVID-19), 2020a, Prime Minister's statement on coronavirus (COVID-19), 2020b). "Self-report period covered" are the time periods we asked participants to consider when making their responses to the questionnaire. "Step count period covered" is the time period over which daily step-counts were analysed for that period.

\begin{tabular}{|c|c|c|c|}
\hline Period label & Date range & Self-report period covered & Step count period covered \\
\hline Pre-restrictions (Baseline) & Prior to 23 March 2020 & Week before 16 March 2020 & February $1^{\text {st }}$ to 29 February 2020 \\
\hline Lockdown (Phase 1) & $\begin{array}{l}23^{\text {rd }} \text { March to } 12 \text { May } \\
2020\end{array}$ & A typical week between $23^{\text {rd }}$ March - 12 May 2020 & $23^{\text {rd }}$ March to 12 May 2020 \\
\hline $\begin{array}{l}\text { Relaxed Restrictions (Phase } \\
\text { 2) }\end{array}$ & 13 May 2020 onwards & $\begin{array}{l}\text { Week prior to survey completion date }\left(29^{\text {th }} \text { May to } 10 \text { June }\right. \\
\text { 2020) }\end{array}$ & $\begin{array}{l}13 \text { May } 2020 \text { to survey completion } \\
\text { date. }\end{array}$ \\
\hline
\end{tabular}


2020 (Table 1). Participants were asked to think about their typical routine, based on the survey period the question referred to. The primary dependent variable we used for analysis was based on self-reported change in PA during Phase 1 and Phase 2 , relative to the Pre-restriction period. This was based on a Likert scale of -5 (substantially reduced) through to +5 (substantially increased), with a zero value relating to no change.

\section{Step count data}

Historic step count data recorded by the Sweatcoin app (Derlyatka et al., 2019), was provided by a subset of participants between 1 February 2020 and the date of survey completion. Step count data with more than $50 \%$ of days with missing stepcount values, were removed. The data was split into three time periods, similar to the survey (see Table 1).

Within each period, daily step count data was averaged across days of the week, resulting in seven mean daily stepcount values (Sunday-Saturday) for each participant, per period. To measure proportional change in step-count during the phase 1 period of restrictions, we divided the mean daily step counts in phase 1 by the corresponding value in the baseline period. The natural log of the resulting values was calculated and then the mean taken to get a final phase 1, log-percentage change for each participant. The same procedure was applied to the phase 2 data to get a corresponding value for this period.

\section{Analyses}

All analyses were completed using the $\mathrm{R}$ programming language (v3.6.2; (R Core Team, 2019)). Multiple regression models were used to analyse the factors associated with PA change during the lockdown period. We investigated both selfreported (self-reported change in PA) and objective (logpercentage change in mean daily step count) measures of change as dependant variables, with the survey data used as predictor variables. Continuous variables were standardised, by mean-centring and scaling by the standard deviation. All independent variables were entered into the regression models simultaneously. Significant variables were defined as $p<0.05$. All regression coefficients are reported with 95\% Confidence Intervals $(\mathrm{Cl})$ in brackets. Multicollinearity was tested for between variables using the variance inflation factors (VIF) method; we report the maximum value $\left(\mathrm{VIF}_{\max }\right)$ from the variables used in the regressions, with a $\mathrm{VIF}_{\text {max }}<5$, classed as an acceptable level of correlation (Daoud, 2017). The models were applied to changes in Phases 1 and 2, relative to baseline periods. For significant categorical variables we plot the mean values for all variables within a category to add further context to the results.

In addition to understanding change in levels of PA, we also investigated how PA routines had changed. This was achieved through a Sankey network of the main types of PA (e.g., running, gym, outdoor sports) respondents participated in across the three periods analysed. We subsequently, analysed the intention to stick with new (or old) routines post-lockdown, for each sport type.

\section{Results}

A table of demographic data for the full sample $(\mathrm{N}=1656)$ and the sub-sample who provided step-count data $(\mathrm{N}=487)$ is provided in Table 2. It should be noted that the age of our

Table 2. Demographic breakdown of the full sample and the sub-sample of participants who provided step-count data. Entries with super-scripted (a) denotes the reference category for that variable used in the regression models.

\begin{tabular}{|c|c|c|}
\hline Variable & $\begin{array}{c}\text { Full sample } \\
(\mathrm{N}=1656) \\
\text { Frequency (\%) }\end{array}$ & $\begin{array}{c}\text { Step-count sample } \\
(\mathrm{N}=487) \\
\text { Frequency }(\%)\end{array}$ \\
\hline \multicolumn{3}{|l|}{ Gender } \\
\hline Male & 48.8 & 42.7 \\
\hline Female $^{a}$ & 50.8 & 56.7 \\
\hline Other & 0.4 & 0.6 \\
\hline \multicolumn{3}{|l|}{ Age (years) } \\
\hline $18-24$ & 37.3 & 41.1 \\
\hline $25-34$ & 30.3 & 30.8 \\
\hline $35-44$ & 17.1 & 18.1 \\
\hline $45-54$ & 9.6 & 8.2 \\
\hline 55 or over & 5.7 & 1.8 \\
\hline \multicolumn{3}{|l|}{ Ethnicity } \\
\hline Black, Asian or minority ethnic & 23.4 & 30.8 \\
\hline White British ${ }^{\mathrm{a}}$ & 76.6 & 69.2 \\
\hline \multicolumn{3}{|c|}{ Weight status (based on body mass index estimate) } \\
\hline Underweight & 5.3 & 4.1 \\
\hline Healthy weight $^{a}$ & 49.3 & 51.1 \\
\hline Overweight & 27.5 & 28.8 \\
\hline Obese & 17.9 & 16.0 \\
\hline \multicolumn{3}{|l|}{ Home location } \\
\hline Rural & 16.9 & 17.9 \\
\hline Suburban & 48.0 & 46.0 \\
\hline Urban $^{\mathrm{a}}$ & 35.1 & 36.1 \\
\hline \multicolumn{3}{|l|}{ Has access to a garden } \\
\hline Yes & 82.7 & 82.8 \\
\hline No & 17.3 & 17.2 \\
\hline \multicolumn{3}{|l|}{ Has dependent children } \\
\hline Yes & 34.2 & 35.5 \\
\hline No & 65.8 & 64.5 \\
\hline \multicolumn{3}{|l|}{ Employment status } \\
\hline Not in employment & 16.4 & 11.1 \\
\hline Student & 14.7 & 15.2 \\
\hline Working from home & 30.5 & 27.9 \\
\hline Working in usual location ${ }^{a}$ & 17.9 & 27.5 \\
\hline Furloughed & 17.6 & 17.9 \\
\hline Retired & 2.9 & 0.4 \\
\hline \multicolumn{3}{|l|}{ COVID status } \\
\hline Believe or definitely had COVID-19 & 11.2 & 14.0 \\
\hline $\begin{array}{l}\text { Believe or definitely not had COVID- } \\
19^{\mathrm{a}}\end{array}$ & 70.8 & 64.5 \\
\hline Unsure & 18.0 & 21.5 \\
\hline \multicolumn{3}{|l|}{ Shielding status } \\
\hline Not shielding ${ }^{a}$ & 93.5 & 92.6 \\
\hline Shielding and adhering & 3.3 & 3.3 \\
\hline Shielding, but not adhering & 2.1 & 3.5 \\
\hline Unsure & 1.1 & 0.6 \\
\hline \multicolumn{3}{|c|}{ Primary form of physical activity prior to lockdown } \\
\hline Walking $^{\mathrm{a}}$ & 36.5 & 33.7 \\
\hline Running/Cycling & 12.5 & 17.2 \\
\hline Team sports & 5.4 & 6.6 \\
\hline Gym & 17.6 & 20.1 \\
\hline Sports classes & 2.9 & 1.8 \\
\hline Home floor exercises & 8.4 & 8.0 \\
\hline $\begin{array}{l}\text { Home machine-based (e.g., exercise } \\
\text { bike, treadmill) }\end{array}$ & 1.8 & 2.5 \\
\hline No routine & 11.6 & 7.2 \\
\hline Other & 3.3 & 2.9 \\
\hline
\end{tabular}


sample was heavily dominated by young adults and wasn't representative of the distribution of age across the UK population.

\section{Self-reported change in PA}

Self-reported change in PA during lockdown Phase 1 was, on average, slightly negative $(\mathrm{M}=-0.30, \mathrm{sd}=2.67, \mathrm{t}=-4.58, \mathrm{p}$ $<0.001$ ). However, the distribution of responses was spread widely, highlighting an almost equal split between those who reported a reduction in PA levels (46.0\%) and an increase in PA levels (39.9\%), with $14.1 \%$ reporting no change. For lockdown Phase 2, there was a significant increase compared to Phase 1 $(M=0.09, s d=2.55$, paired $t=7.54, p<.001)$, although the mean did not significantly differ from zero $(t=1.50, p=0.135)$. This was reflected in the distribution with more people reporting an increase $(43.7 \%)$ or no change $(18.3 \%)$ in PA levels compared to the pre-lockdown periods, with $38.0 \%$ reporting a decrease.

\section{Change in step count}

Prior to the lockdown periods, mean daily step count across the sample ( $\mathrm{N}=487$ ) was 6680.53 ( $\mathrm{sd}=3310.24)$. The lockdown phases had a significant impact on mean daily step count in contrast to the pre-lockdown period $(F(1.61,781.94)=72.84, p$ $<0.001$ ), with the mean daily number of steps reducing to a mean of 5157.07 ( $\mathrm{sd}=3474.58$ ) in Phase 1. In Phase 2, mean daily step-count was 6197.62 ( $\mathrm{sd}=4028.07$ ), remaining lower than pre-lockdown $(p=0.006)$ but was significantly higher than during Phase $1(p<0.001)$.

\section{Factors associated with self-reported physical activity change}

Factors associated with the self-reported change in PA for both Phase 1 and Phase 2 lockdowns, relative to the Baseline period are shown in Figure $1\left(\mathrm{VIF}_{\text {max }}=2.8\right.$; Phase $1: \mathrm{N}=1656, \mathrm{R}^{2}=0.12$; Phase $2: N=1656, R^{2}=0.13$ ). To improve clarity, categories in which no variables were significant are omitted from the figure. The full table of results for all independent variables is provided in Supplementary Information A.

\section{Work status}

People who were on furlough from work showed a positive relationship with self-reported change in PA in Phase 2 only (Figure $1 ; b=0.48(0.07,0.89), p=0.020)$.

\section{Personality}

There was a significant positive relationship between the independent variable, Extroversion (on the Big-Five personality scale) and self-reported change in PA in both Phase 1 (Figure $1 ; b=0.20(0.063,0.34, p=0.005)$ and Phase $2(b=0.17(0.033$, $0.31), p=0.014)$.

\section{Demographics}

There was a significant negative relationship between the independent variable age and self-reported change in PA in Phase 1 (Figure $1 ; b=-0.24(-0.40,-0.08), p=0.003)$ and Phase $2(b=-0.21(-0.35,-0.07), p=0.005)$. In addition, we found that the rewards app users (i.e., the sample of respondents collected through the Sweatcoin app) showed a positive relationship with self-reported change in PA in Phase 2 (Figure $1 ; b$ $=0.30(0.05,0.56), p=0.027)$, relative to the respondents from the survey panel.

\section{Wellbeing}

We noted a positive relationship between self-reported change in PA and the Happiness rating from the Office of National Statistics wellbeing scale (Waldron, 2010) in both Phase 1 (Figure $1 ; b=0.30(0.10,0.50), p=0.003)$ and Phase 2 $(b=0.38(0.18,0.58), p<0.001)$. In addition, the general health rating had a positive relationship with self-reported change in PA in Phase 2 (Figure 1; $b=0.23(0.09,0.37), p=0.002)$.

\section{Residence}

Residents in rural locations showed a significant positive association with self-reported change in PA, during both Phase 1 (Figure $1 ; b=0.87(0.50,1.24), p<0.001)$ and Phase $2(b=0.61$ $(0.24,0.98), p=0.001)$. Those in suburban residences showed a positive association with self-reported change in PA in Phase 2 only (Figure $1 ; b=0.41(0.12,0.70), p=0.006)$. Plots of mean self-reported change in PA by residence (Figure $2 a$ ), highlights that in comparison to urban residents, who reported reduced levels of PA in both phases, rural residents reported increased PA.

\section{Body Mass Index (BMI) classification}

People classed as obese had a significant negative relationship with self-reported change in PA in Phase 1 (Figure $1 ; b=-0.51$ $(-0.88,-0.14), p=0.008)$. Plots of mean self-reported change in PA by BMI classification show that all age groups reported reductions in self-reported PA during Phase 1 (Figure 2b). However, in comparison to people of a healthy weight, those classed as obese reported a substantially greater decrease in PA.

\section{Pre-lockdown primary activity type}

Respondents whose primary activity was running or cycling prior to lockdown showed a significant positive relationship with self-reported change in PA in both Phase 1 (Figure $1 ; \mathrm{b}$ $=0.57(0.16,0.98), p=0.006)$ and Phase $2(b=0.46(0.11,0.81), p$ $=0.012$ ). In contrast, those who primarily attended the gym prior to lockdown showed a significant negative relationship to self-reported change in PA in both Phase 1 (Figure $1 ; b=-1.10$ $(-1.49,-0.71), p<0.001)$ and Phase $2(b=-1.45(-1.82,-1.08)$, $p<0.001)$. Plots of mean self-reported change in PA by prelockdown primary activity type show that compared to those whose activity was walking, gym users reported substantial reductions in self-reported PA throughout the lockdown periods (Figure 2c); whilst runners/cyclists reported increased levels of PA.

\section{Factors associated with step-count change}

A further multiple regression model was run on the subset of participants who had provided step-count data. We used the logpercentage change in step count for both Phase 1 and Phase 2 lockdown periods, relative to the Baseline period (Table 1) as the 


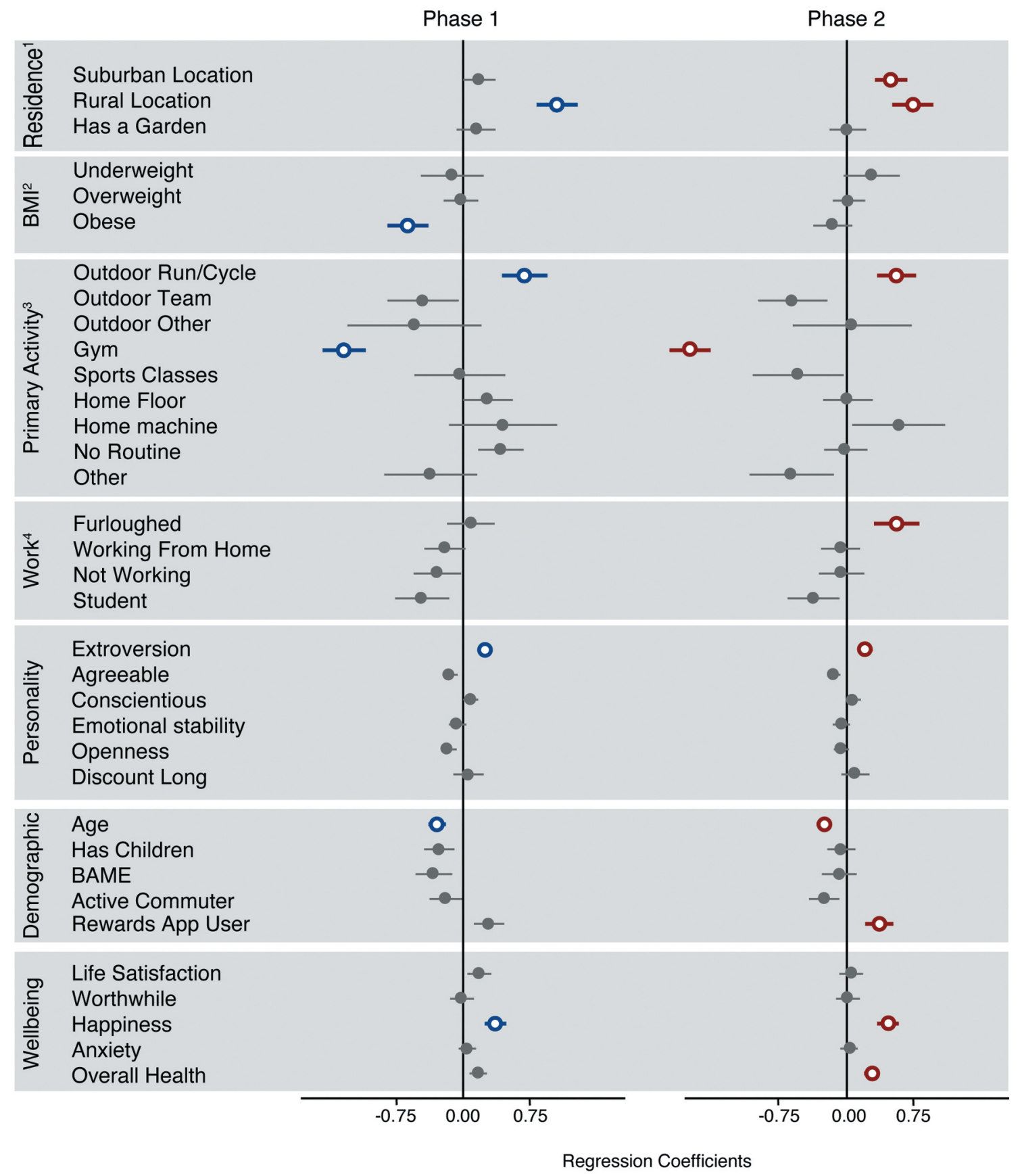

Figure 1. Plot of regression coefficients for the multiple regression model of self-reported change in physical activity relative to the Baseline period. Significant factors are highlighted in thicker line with open circle marker in blue for Phase 1 and red for Phase 2 lockdown periods. Non-significant factors are shown as grey filled circle markers. Error bars represent standard errors. (1) Residence category coefficients are shown relative to Urban; (2) Body Mass Index (BMI) category coefficients are shown relative to Healthy Weight; (3) Primary Activity category coefficients are shown relative to Walking; (4) Work category coefficients are shown relative to Working as Usual.

dependent variable. The factors associated with change in step count $\left(\mathrm{VIF}_{\max }=2.9\right.$; Phase 1: $\mathrm{N}=487, \mathrm{R}^{2}=0.27$; Phase 2: $\mathrm{N}=487$, $\left.R^{2}=0.25\right)$ are shown in Figure 3. To improve clarity, categories in which no variables were significant are omitted from the Figure. For full table of results see Supplementary information B.

\section{Body Mass Index (BMI) Classification}

In contrast to those classed as healthy weight, people classed as obese had a significant negative relationship with change in step count in Phase 1 only (Figure 3; $b=-0.21(-0.37,-0.05), p$ $=0.009$ ). 
a

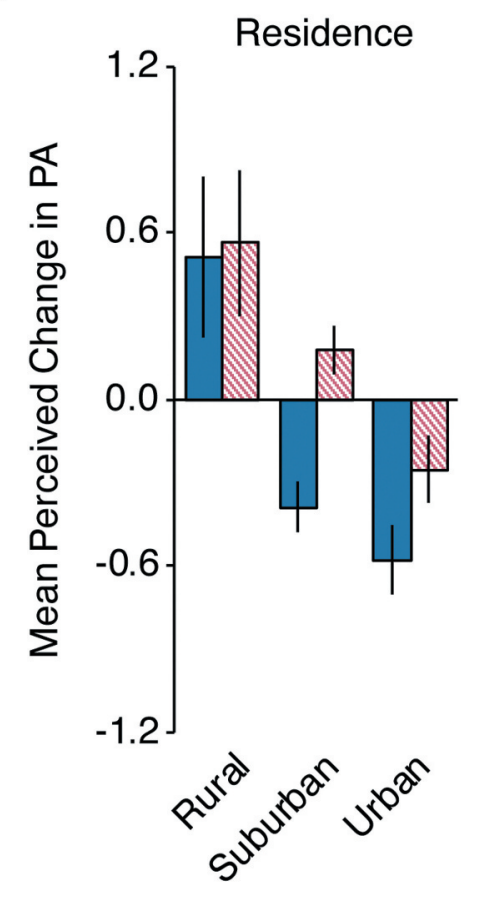

b

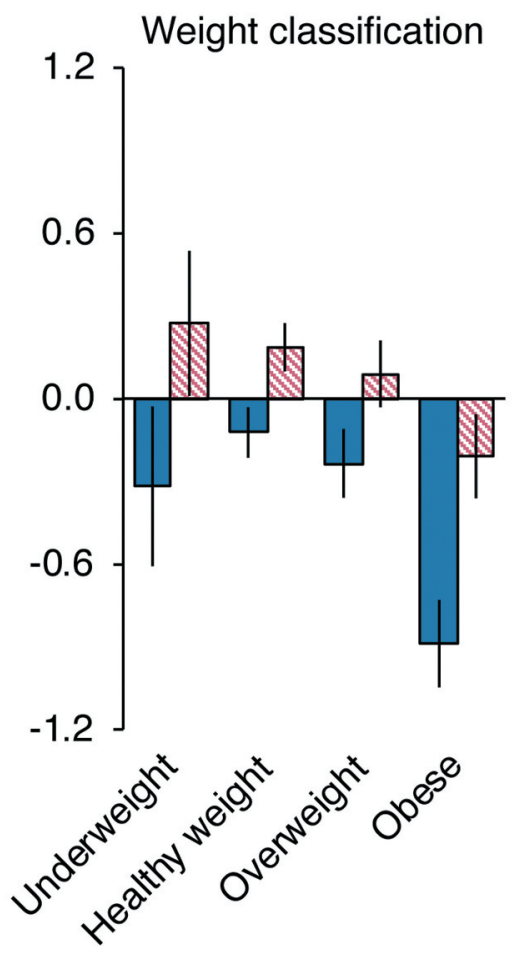

C

Primary form of physical activity

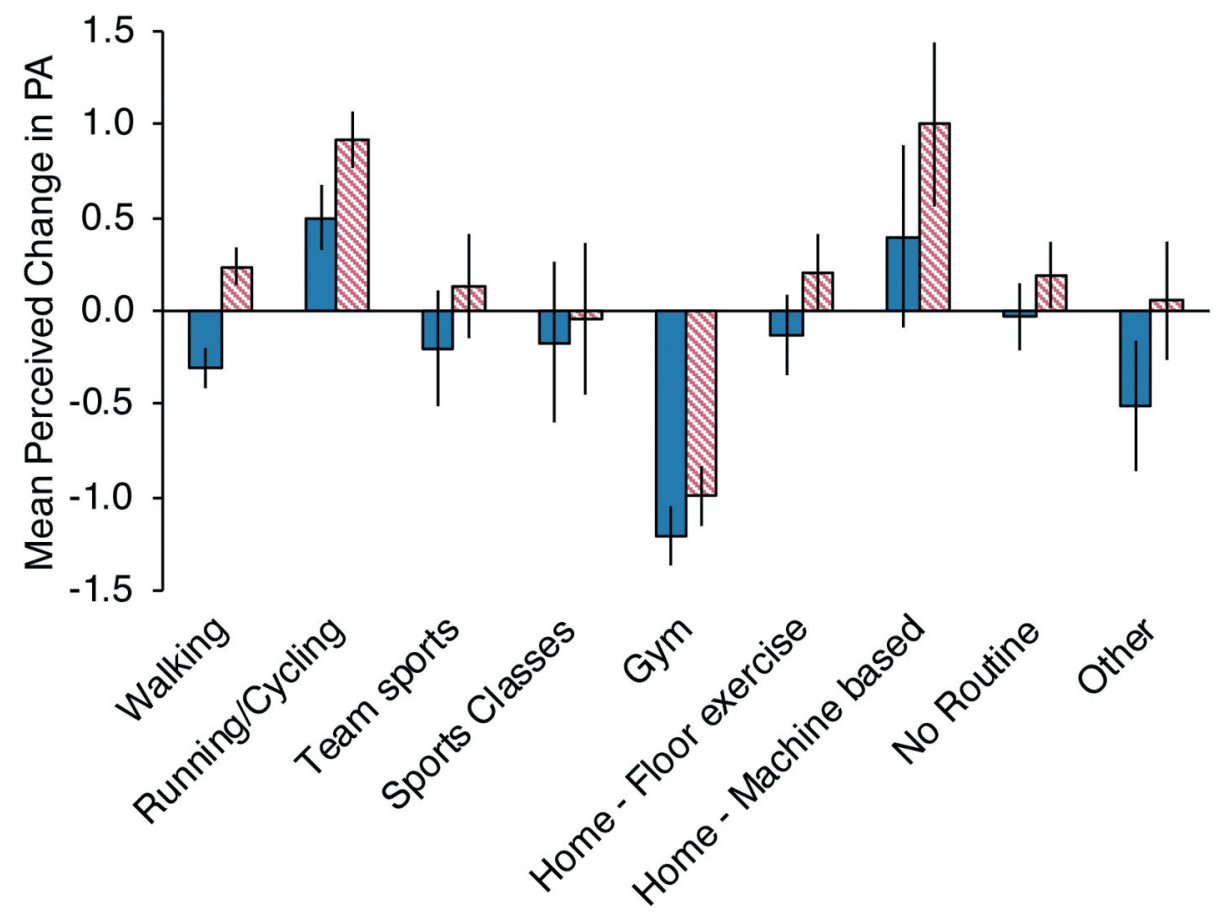

Figure 2. Mean values of self-reported change in physical activity, by residential location (A), weight classification (B), and primary PA type prior to restrictions (C). Based on a Likert-scale between +5 (substantial increase) and -5 (substantial decrease). Solid blue bars represent Phase 1 period, pink-hatched bars represent Phase 2 period. Error bars represent standard error of the mean. 


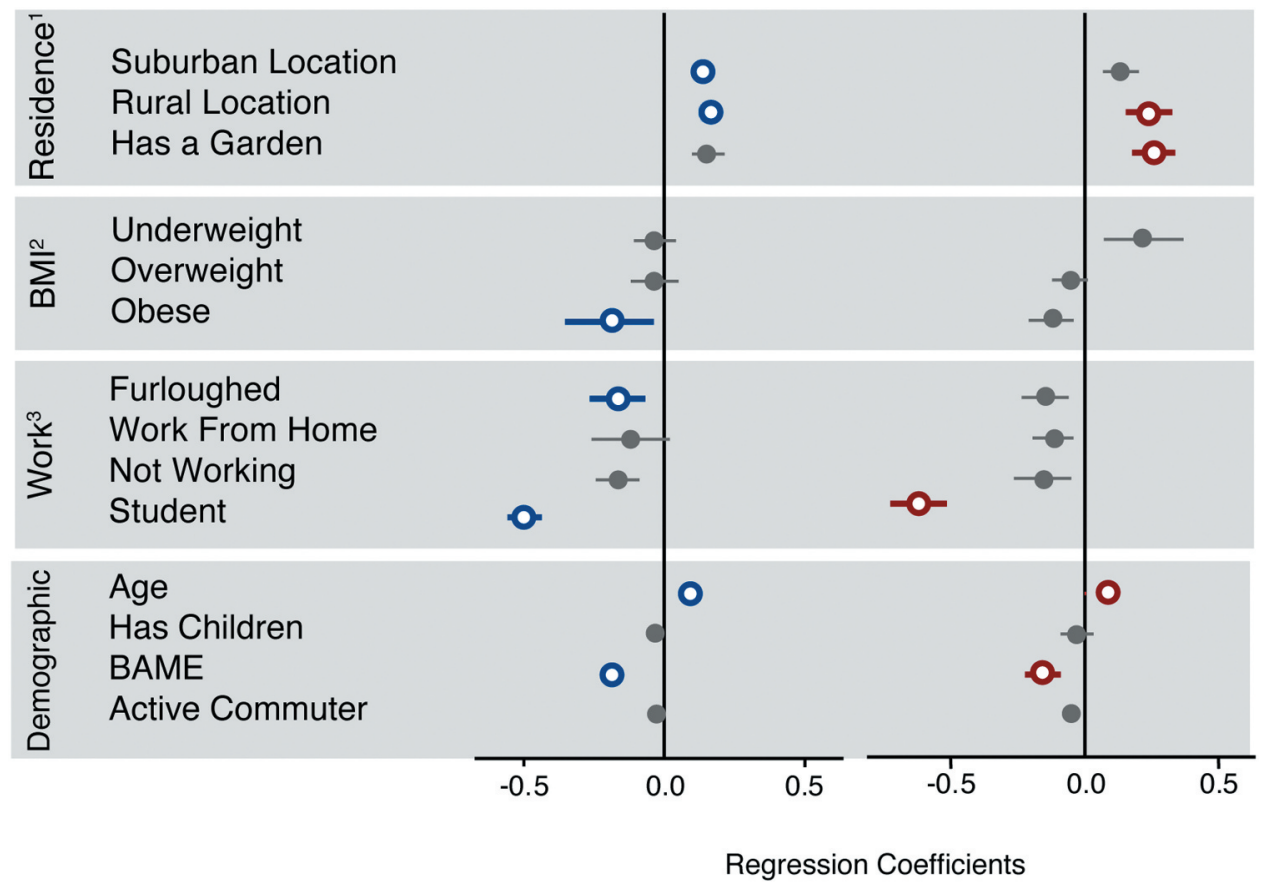

Figure 3. Plot of regression coefficients for the multiple regression model of log-percentage change in mean daily step count relative to the Baseline period. Significant factors are highlighted in thicker line with open circle marker in blue for Phase 1 and red for Phase 2 lockdown periods. Non-significant factors are shown as grey filled circle markers. Error bars represent standard errors. (1) Residence category coefficients are shown relative to Urban; (2) Body Mass Index (BMI) category coefficients are shown relative to Healthy Weight; (3) Work category coefficients are shown relative to Working as Usual.

\section{Residence}

Residents in rural locations showed a significant positive relation to change in step-count, during both Phase 1 (Figure $3 ; \mathrm{b}$ $=0.18(0.02,0.34), p=0.022)$ and Phase $2(b=0.24(0.06,0.42), p$ $=0.008)$. Those in suburban residences showed a positive relation in Phase 1 only (Figure 3; $b=0.15(0.03,0.27), p=0.015)$, while those who had gardens showed a positive relation to change in step count during Phase 2 only (Figure $3 ; b=0.26$ $(0.08,0.44), p=0.004)$. Plots of mean log-percentage change in step count showed urban residents (the reference variable) reported the largest reduction (Figure 4a) compared to other groups.

\section{Work status}

People who were on furlough from work showed a negative relationship with change in step count during Phase 1 (Figure 3; $\mathrm{b}=-0.17(-0.33,-0.01), \mathrm{p}=0.041)$. In addition, students also showed negative relationships in both Phase 1 (Figure $3 ; \mathrm{b}$ $=-0.52(-0.72,-0.32), p<0.001)$ and Phase $2(b=-0.62$ $(-0.86,-0.38), p<0.001)$. Plots of mean log-percentage change in step count (Figure $4 b$ ) showed that students had the largest reduction across both Phase 1 and 2 .

\section{Demographics}

There was a significant positive relationship between age and change in step count in Phase 1 (Figure $3 ; b=0.11(0.05,0.17), p$ $<0.001)$ and Phase $2(b=0.09(0.03,0.15), p=0.006)$. In addition, we found that for the ethnicity category, Black, Asian and minority ethnic (BAME) groups showed a significant negative relationship with change in step count in Phase 1
(Figure 3; $b=-0.18(-0.32,-0.04), p=0.008)$ and Phase 2 $(b=-0.15(-0.31,-0.01), p=0.049)$. The plots of mean logpercentage change in step count (Figure 4c) highlight the substantial reduction in step-count during the lockdown period in BAME groups in contrast to White British respondents.

\section{Intentions to stick to new or old routines post-lockdown}

The main exercise activities of $25.9 \%$ of the sample became restricted during the Phase 1 lockdown (i.e., gyms and fitness classes closed, outdoor teams sports not allowed). Of the remainder, $62.5 \%$ took part in activities that weren't subsequently restricted (i.e., home exercises and outdoor walking, running or cycling) and $11.6 \%$ had no routine prior to the restrictions.

The proportion of those participating in unrestricted activities increased to $83.3 \%$ and $85.1 \%$ in Phases 1 and 2, respectively. However, there was also a small increase in those reporting no specific PA routine during Phase 1 (14.1\%) and Phase 2 (12.5\%). The changes in routine are further visualised in the Sankey diagram (Figure 5).

Finally, we asked those who had changed to a new routine, due to their previous primary activity being restricted, whether they planned to stick to it (Table 3).

\section{Discussion}

Overall, we found that average step count, measured objectively from smartphone data, reduced during both phases of lockdown in comparison to the period in February prior to the lockdown periods. Taking into account seasonality, this 
a

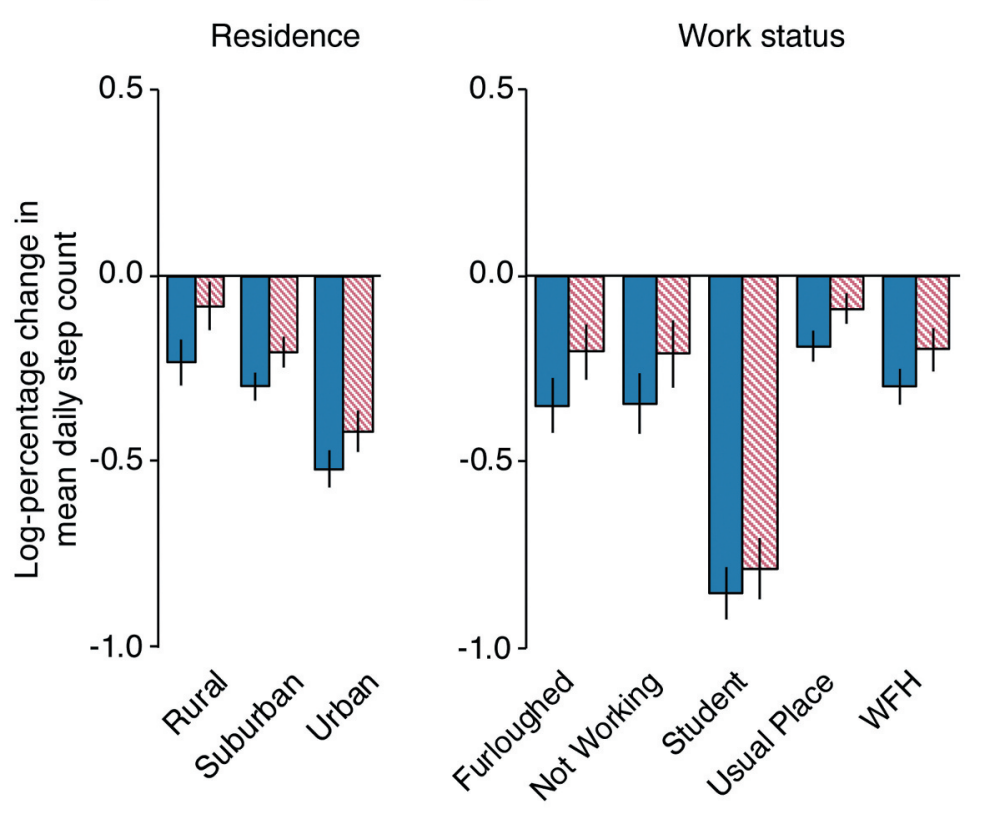

C

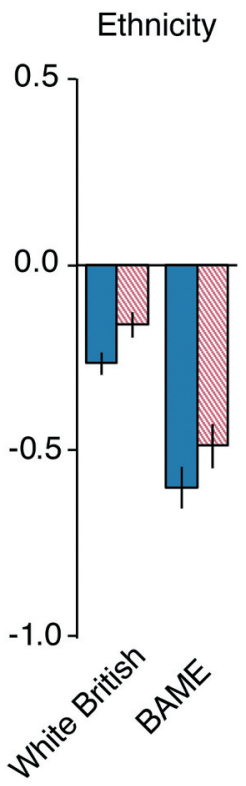

Figure 4. Log-percentage change in mean daily step count for residential location (A), work status (B), and ethnicity (C). Solid blue bars represent Phase 1 period, pinkhatched bars represent Phase 2 period. Error bars represent standard error of the mean.

reduction is even more substantial as typically step-count would rise through the months of March-May, when the weather becomes more favourable (Tucker \& Gilliland, 2007; Tudor-Locke et al., 2004). Similar results have been reported internationally from other app-based measures of step-count recently (Tison et al., 2020), corroborating the impact lockdown had on activity levels. Here we have provided a more detailed insight using a comprehensive questionnaire in parallel with the step-count data from a large sample to understand which groups have shown the greatest reductions.

While step-count provides a useful objective indicator of PA levels, it must be recognised this only captures a single modality of activity. Therefore, we further captured self-reported change in PA levels from respondents. This self-reported data also provided a larger sample for analysis. Importantly, the
Baseline

Phase 1

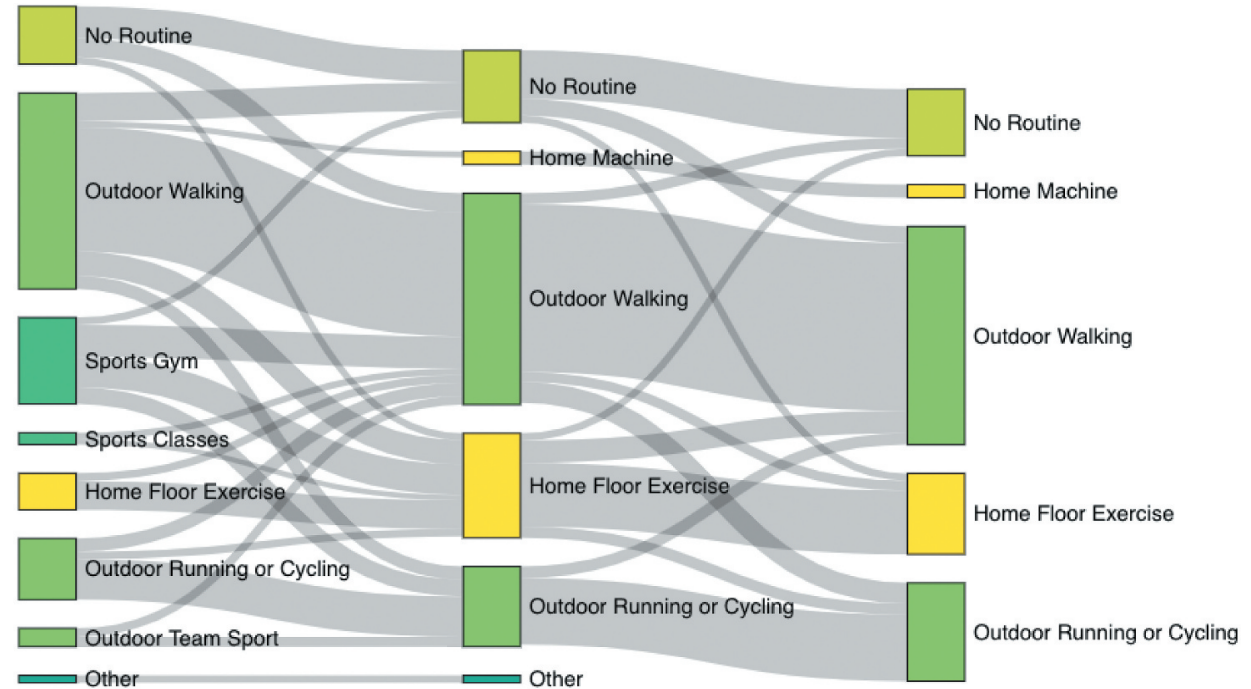

Figure 5. Sankey diagram showing the switch of main physical activity type across the lockdown periods. Block sizes represent proportion of the sample undertaking the activity type. To increase clarity, counts of $<15$ are not displayed on the diagram. 
Table 3. Proportion of respondents who stated they were likely to stick with their new routine once restrictions were lifted or return to their old routine. The results are grouped by the activity participants stated as their primary activity prior to lockdown. These are sub-grouped into activity that were subsequently restricted or remained unrestricted.

\begin{tabular}{|c|c|c|c|c|}
\hline & $\begin{array}{c}\text { Activity Type } \\
\text { (pre- } \\
\text { lockdown) }\end{array}$ & $\begin{array}{l}\text { Stick to new } \\
\text { routine } \\
\text { (percentage) }\end{array}$ & $\begin{array}{l}\text { Stick to old } \\
\text { routine } \\
\text { (percentage) }\end{array}$ & $\begin{array}{c}\text { Unsure } \\
\text { (percentage) }\end{array}$ \\
\hline \multirow{3}{*}{$\begin{array}{l}\text { Restricted } \\
\text { during } \\
\text { lockdown }\end{array}$} & Gym & 31.2 & 63.5 & 5.3 \\
\hline & $\begin{array}{l}\text { Fitness } \\
\text { Classes }\end{array}$ & 28.6 & 66.7 & 4.8 \\
\hline & $\begin{array}{c}\text { Outdoor } \\
\text { Team } \\
\text { Sports }\end{array}$ & 43.0 & 44.3 & 12.7 \\
\hline No routine & No Routine & 58.3 & 16.7 & 25.0 \\
\hline \multirow{5}{*}{$\begin{array}{l}\text { Un-restricted } \\
\text { during } \\
\text { lockdown }\end{array}$} & $\begin{array}{c}\text { Home Floor } \\
\text { Exercises }\end{array}$ & 43.2 & 38.4 & 18.4 \\
\hline & $\begin{array}{l}\text { Home } \\
\text { Machine } \\
\text { Exercises }\end{array}$ & 37.0 & 44.4 & 18.5 \\
\hline & $\begin{array}{l}\text { Outdoor } \\
\text { Running/ } \\
\text { Cycling }\end{array}$ & 53.4 & 35.3 & 11.3 \\
\hline & Walking & 43.2 & 37.5 & 19.4 \\
\hline & Other & 27.3 & 70.9 & 1.8 \\
\hline
\end{tabular}

distribution of responses differed to that resulting from stepcount analyses, with a mean value close to zero in both periods of lockdown. This highlighted a clear split, between those who considered their levels of PA had increased during the lockdown periods, and those who considered it had decreased. Furthermore, we found a number of differing and contrasting significant factors associated with step-count compared to the self-reported change in PA. This included age being positive in the step-count regression, but negative for self-reported change in exercise and similarly, people who were furloughed having a negative relationship to step count change, but a positive relationship with self-reported change in PA. This highlights that objective and self-reported measures are not necessarily correlated and may capture different aspects of PA. For example, those who switch to more outdoor activities such as walking are likely to show increased step-count, but may feel this is less-physically intensive than their previous activity - e.g., using weights in the gym, which wouldn't be captured by the pedometer in a smartphone.

The subsequent analyses have highlighted the stark contrasts within groups defined by the demographic, lifestyle and health factors associated with increases or decreases in PA during the UK lockdown periods. These are discussed in more detail below.

\section{Residential environment}

One of the factors that was significant for both self-reported and objective measures of PA was the residential location of participants; those living in rural and suburban locations showed a perceived increase in PA and a lower reduction in step count in at least one lockdown phase. In contrast, urban residents reported a reduction in self-reported PA as well as step count in both phases. The restriction to all but essential travel and closure of sports/gym facilities resulted in highly localised PA options (McDougall et al., 2020). This has emphasised inequalities between rural locations with open green space and urban environments with limited green space and poor walking infrastructure (McCormack et al., 2004), that cannot support localised PA (McDougall et al., 2020).

\section{Health factors}

A concerning finding was that those classed as obese, and hence already likely to have sedentary lifestyles, were reporting substantially lower levels of exercise than those in other weight groups. While there was no significant difference to other weight groups in percentage step-count reduction, the obese group also reported an overall reduction in step-count during both phases. It has been identified that those classed as obese are at higher risk of developing complications from COVID-19 (Kimura \& Namkoong, 2020; Lighter et al., 2020), with the impact of reduced PA on the immune system being a contributing factor (Kimura \& Namkoong, 2020). Hence, it is concerning that lockdown restrictions could potentially exacerbate this group's vulnerability due to further reductions in PA in an already inactive group.

In addition to physical health, PA is positively associated with mental health (Edwards \& Loprinzi, 2016; Ginoux et al., 2021). It is noteworthy therefore, that there was a significant positive correlation between self-reported change in PA and the happiness rating from the ONS4 scale. This highlights, and further corroborates similar studies (e.g., (Ginoux et al., 2021)) that, on average, those who had increased PA during lockdown were also more likely to be happier during that period. However, given the model we have used, we can't infer the directionality of this relationship. There is evidence however, that a sudden stop in PA in previously active people risks increasing depressive symptoms within a short period of time (Edwards \& Loprinzi, 2016). Hence, the sudden reduction in activity levels, from those who have been unable to maintain their usual routine may have exacerbated this relationship between mood and change in activity, which has been shown to have deteriorated nationally in the UK during the lockdown (Pierce et al., 2020).

\section{Ethnicity}

Another group, evidenced to be at higher risk from COVID-19 are those from BAME populations (Bhatia, 2020; Niedzwiedz, O'Donnell, Jani et al., 2020). Again, we found a stark contrast in PA levels, in terms of reduced step-count, in those from BAME groups compared to those identifying as White British.

Age

We observed a contrast of age within the analyses, with a negative correlation of age with self-reported change in PA, in line with other research (Rogers et al., 2020), versus a positive correlation of age with step-count. From this we can infer that older age groups feel that their overall PA levels have reduced more in comparison to younger groups during the lockdown periods. However, older groups were possibly more likely to switch to walking or running activities resulting in a smaller reduction in step count than younger groups, also mirrored by the significant reduction in students' step-count compared to 
other work groups. Given the sample demographic however, it is important to contextualise these results by highlighting that older groups here are more likely to be defined as middle-aged adults, as opposed to older adults per se $(<6 \%$ of the sample were aged over 55 years).

\section{Personality}

We found that people scoring highly on Extraversion were associated positively with self-reported change in PA. This aligns with the literature studying the relationships between PA and personality, where Extraversion is likely to be associated with individuals who are more physically active. Moreover, Extraversion (and Consciousness) have been suggested to be positively related with exercise intention-behaviour (Hoyt et al., 2009), with these groups possibly being more driven to find alternative methods of PA over a shorter period of time following the restrictions.

\section{Exercise types}

The primary form of PA (prior to restrictions) impacted selfreported change in PA during the lockdown periods. Those who were primarily runners/cyclists tended to report increased levels of activity during the restrictions, possibly having more opportunities to undertake this opportunity. Gym users reported by far the biggest reduction in self-reported PA during the restrictions. This highlights the reliance and habituation gym users have on these facilities, which were closed during the lockdown periods. It is clear that, whilst most switched to new outdoor or home-based activities during the closures, they did not feel they were achieving the same level of exercise as their previous routines. This is further reflected in the fact that two-thirds of gym users planned to return to their previous PA routines, once restrictions were lifted. A similar proportion planned to return to fitness classes, and highlights the strong reliance and affiliation to these types of PA. A study of how the change of context to PA due to the lockdown periods affected habits, supports these findings (Maltagliati et al., 2020). The study found that although PA habits were weakened at the start of lockdown, individuals were able to "renegotiate or develop new PA habits" in the mid-end stages of lockdown.

The lockdown period did provide some opportunity to those who previously reported having no specific PA routine. Of those who developed a routine during the restrictions, over half planned to continue with this new routine once restrictions had lifted.

\section{Restriction phase}

We captured results for both Phase 1 restrictions where all sports facilities were close and people were limited to one period of outdoor exercise per day, and Phase 2 restrictions where outdoor exercise was no longer limited, but sports facilities remained closed. Our results indicated that the relaxation of restrictions on outdoor exercise had a positive effect, with an overall increase in mean daily step count, compared to Phase 1. In particular, individuals on Furlough or who were obese were significantly associated with negative change in step-count for
Phase 1 only, suggesting these groups increased their stepcounts in Phase 2 relative to Phase 1 . However, overall the mean daily step-count in Phase 2 remained lower than before restrictions were put in place.

We saw a similar result for the self-reported change in PA results, with the proportion of the sample reporting a reduction in perceived PA levels reducing from $46 \%$ in Phase 1 to $38 \%$ in Phase 2. The breakdown of groups (Figure 2) highlights the overall change in perceived PA levels in Phase, with those living in suburban areas switching to a significantly positive association in Phase 2. People classed as obese also went from a significant negative association in Phase 1, to no significant association in Phase 2. Again, this suggests the opportunity for unlimited outdoor exercise had a positive impact on some, although in addition, we can also consider that over time people may have settled into finding other alternative exercise options, compared to the early stages of lockdown (Maltagliati et al., 2020).

\section{Limitations}

The sample of respondents lacked older adults - less than $6 \%$ of the full sample were over the age of 55 years. Therefore, we cannot generalise our results to older age groups. However, the large sample we collected did allow us to provide a comprehensive insight into how the pandemic related restrictions have impacted PA across different demographic groups.

As with all self-report scales, the self-reported change in PA was subject to people retrospectively recalling their perception of PA levels prior to and during the lockdown phases, meaning this measure to be more a "perceived" status (Cross et al., 2021). However, the time periods were relatively short, and the abruptness of change when restrictions were introduced are likely to have resulted in a clear perception of how an individual had changed their behaviour. Related to this we captured some variables based on the present time (e.g., the ONS4 wellbeing questions), reducing the confidence in any causal relationship between these and the time-based variables. However, to counteract the limitations of self-reported measures, we have combined them with step-count measures recorded from participants smartphones, which has provided a complementary and objective assessment of PA change both prior to and during the lockdown periods.

Finally, we recommend that further studies in this area could consider stratifying groups according to their level of motivation to exercise, to determine how motivation could moderate changes in PA due to restrictions. It is worth noting from our results, that the sample of users of the Sweatcoin rewards app were more positively associated with change in self-reported PA, compared to the sample from the general survey panel. This suggests that incentivising PA still had some positive effect during restrictions, in a similar way to that reported in normal circumstances (Elliott et al., 2019; Lemola et al., 2021).

\section{Conclusion}

The results from the study highlight the dichotomy the impact has had on PA routines. Crucially, groups at high risk of complications from COVID-19 appear to be also impacted in terms of 
substantial reduction in PA. More specifically, those who are obese are at risk of further reducing already low activity levels; if the impact of continued restrictions has a long-term effect on routines, this further reduction could become habitualised. Therefore, we suggest that interventions are required to support these groups, to ensure they have access and motivation to participate in physical activities, whether this is home based or outdoors. In addition, we have observed stark contrasts between those living in urban versus rural locations, emphasising the need for better urban design and planning that facilitates safe and accessible environment for outdoor PA.

On the other hand, we have seen some groups develop new routines and increase (self-reported) levels of PA during the restrictions. Support should also be provided to these groups to maintain these new routines to ensure they are long lasting, and hence beneficial to both their mental and physical health.

\section{Author contributions}

MTE, CJ, IV designed the study and collected the data; MTE, VE, MS analysed the data; All authors contributed to writing of the manuscript.

\section{Acknowledgments}

We thank Shaun Azam and the team at Sweatco Ltd, for their assistance with data collection through the Sweatcoin app. We further thank Dr Lukasz Walasek for his assistance with the design and ethical approval applications for this project.

\section{Disclosure statement}

No potential conflict of interest was reported by the author(s)

\section{Funding}

This study was funded by the University of Warwick Global Research Priorities for Health Network. MTE has previously held joint funding (Innovate UK) with Sweatco Ltd, the developers of the Sweatcoin platform. $\mathrm{KJ}$ and IV are part funded by the National Institute for Health Research (NIHR) Applied Research Centre (ARC) West Midlands. The views expressed are those of the author(s) and not necessarily those of the NIHR or the Department of Health and Social Care.

\section{ORCID}

Kate Jolly (iD http://orcid.org/0000-0002-6224-2115

Ivo Vlaev (iD http://orcid.org/0000-0002-3218-0144

Mark T. Elliott (iD) http://orcid.org/0000-0003-4000-0198

\section{Data sharing}

Anonymised questionnaire responses and step count data are available from the OSF respository: https://osf.io/b4wz8.

\section{References}

Bhatia, M. (2020). COVID-19 and BAME Group in the United Kingdom. The International Journal of Community and Social Development, 2(2), 269-272. https://doi.org/10.1177/2516602620937878

Biddle, S. J. H., Mutrie, N., Gorely, T., \& Faulkner, G. (2021). Physical activity and psychological well-being. In Psychology of Physical Activity. Routledge.
Caputo, E. L., \& Reichert, F. F. (2020). Studies of Physical Activity and COVID-19 During the Pandemic: A Scoping Review. Journal of Physical Activity and Health, 17(12), 1275-1284. https://doi.org/10.1123/jpah.2020-0406

Cheval, B., Sivaramakrishnan, H., Maltagliati, S., Fessler, L., Forestier, C., Sarrazin, P., Orsholits, D., Chalabaev, A., Sander, D., Ntoumanis, N., \& Boisgontier, M. P. (2020). Relationships between changes in self-reported physical activity, sedentary behaviour and health during the coronavirus (COVID-19) pandemic in France and Switzerland. Journal of Sports Sciences, 1-6. https://doi.org/10.1080/02640414.2020.1841396

Comparison of furloughed jobs data - Office for National Statistics. https:// www.ons.gov.uk/businessindustryandtrade/business/businessservices/ articles/comparisonoffurloughedjobsdata/maytojuly2020 (accessed 15 December 2020)

Coronavirus and homeworking in the UK - Office for National Statistics. https://www.ons.gov.uk/employmentandlabourmarket/peopleinwork/ employmentandemployeetypes/bulletins/coronavirusandhomeworkin gintheuk/april2020 (accessed 15 December 2020)

Cross, T. J., Isautier, J. M. J., Stamatakis, E., Morris, S. J., Johnson, B. D., Wheatley-Guy, C., \& Taylor, B. J. (2021). Self-reported physical activity before a COVID-19 'lockdown': Is it just a matter of opinion? BMJ Open Sport \& Exercise Medicine, 7(2), e001088. https://doi.org/10.1136/ bmjsem-2021-001088

Daoud, J. I. (2017). Multicollinearity and Regression Analysis. J Phys: Conf Ser, 949(1), p. 012009. https://doi.org/10.1088/1742-6596/949/1/012009

De Raad, B. (2000). The Big Five Personality Factors: The psycholexical approach to personality. Hogrefe \& Huber Publishers.

Derlyatka, A., Fomenko, O., Eck, F., Khmelev, E., \& Elliott, M. T. (2019). Bright spots, physical activity investments that work: Sweatcoin: A steps generated virtual currency for sustained physical activity behaviour change. British Journal of Sports Medicine, 53(18), 1195-1196. https://doi.org/10. 1136/bjsports-2018-099739 bjsports-2018-099739

Di Corrado, D., Magnano, P., Muzii, B., Coco, M., Guarnera, M., De Lucia, S., \& Maldonato, N. M. (2020). Effects of social distancing on psychological state and physical activity routines during the COVID-19 pandemic. Sport Sciences for Health, 16(4), 619-624. https://doi.org/10.1007/ s11332-020-00697-5

Docherty, A. B., Harrison, E. M., Green, C. A., Hardwick, H. E., Pius, R., Norman, L., Holden, K. A., Read, J. M., Dondelinger, F., Carson, G., Merson, L., Lee, J., Plotkin, D., Sigfrid, L., Halpin, S., Jackson, C., Gamble, C., Horby, P. W., Nguyen-Van-Tam, J. S., Ho, A., \& Semple, M. G. (2020). Features of 20133 UK patients in hospital with covid-19 using the ISARIC WHO Clinical Characterisation Protocol: Prospective observational cohort study. BMJ, 369, m1985. https://doi.org/10.1136/bmj.m1985

Dunton, G. F., Do, B., \& Wang, S. D. (2020). Early effects of the COVID-19 pandemic on physical activity and sedentary behavior in children living in the U.S. BMC Public Health, 20(1), 1351. https://doi.org/10.1186/ s12889-020-09429-3

Edwards, M. K., \& Loprinzi, P. D. (2016). Effects of a sedentary behaviorinducing randomized controlled intervention on depression and mood profile in active young adults. Mayo Clinic Proceedings, 91(8), 984-998. https://doi.org/10.1016/j.mayocp.2016.03.021

Elliott, M., Eck, F., Khmelev, E., Derlyatka, A., \& Fomenko, O. (2019). Physical activity behavior change driven by engagement with an incentive-based app: evaluating the impact of Sweatcoin. JMIR mHealth and uHealth, 7(7), e12445. https://doi.org/10.2196/12445

Ginoux, C., Isoard-Gautheur, S., Teran-Escobar, C., Forestier, C., Chalabaev, A., Clavel, A., \& Sarrazin, P. (2021). Being active during the lockdown: the recovery potential of physical activity for well-being. International Journal of Environmental Research and Public Health, 18(4), 1707. https://doi.org/10.3390/ijerph18041707

Gosling, S. D., Rentfrow, P. J., \& Swann, W. B. (2003). A very brief measure of the Big-Five personality domains. Journal of Research in Personality, 37 (6), 504-528. https://doi.org/10.1016/S0092-6566(03)00046-1

Harrington, D., \& Hadjiconstantinou, M. (2020). Commuting behaviours and COVID-19. https://doi.org/10.31219/osf.io/46hzd

Hoyt, A. L., Rhodes, R. E., Hausenblas, H. A., \& Giacobbi, P. R. (2009). Integrating five-factor model facet-level traits with the theory of planned behavior and exercise. Psychology of Sport and Exercise, 10 (5), 565-572. https://doi.org/10.1016/j.psychsport.2009.02.008 
Jakobsson, J., Malm, C., Furberg, M., Ekelund, U., \& Svensson, M. (2020). Physical Activity During the Coronavirus (COVID-19) Pandemic: Prevention of a Decline in Metabolic and Immunological Functions. Front Sports Act Living, 2(2), p 57. https://doi.org/10.3389/fspor.2020.00057.

Kimura, T., \& Namkoong, H. (2020). Susceptibility of the obese population to COVID-19. International Journal of Infectious Diseases, 101, 380-381. https://doi.org/10.1016/j.ijid.2020.10.015

Lemola, S., Gkiouleka, A., Read, B., Realo, A., Walasek, L., Tang, N. K. Y., \& Elliott, M. T. (2021). Can a 'rewards-for-exercise app' increase physical activity, subjective well-being and sleep quality? An open-label singlearm trial among university staff with low to moderate physical activity levels. BMC Public Health, 21(1), 782. https://doi.org/10.1186/s12889021-10794-w

Lighter, J., Phillips, M., Hochman, S., Sterling, S., Johnson, D., Francois, F., \& Stachel, A. (2020). Obesity in Patients Younger Than 60 Years Is a Risk Factor for COVID-19 Hospital Admission. Clinical Infectious Diseases, 71 (15), 896-897. https://doi.org/10.1093/cid/ciaa415

Maltagliati, S., Rebar, A., Fessler, L., Forestier, C., Sarrazin, P., Chalabaev, A. Sander, D., Sivaramakrishnan, H., Orsholits, D., Boisgontier, M. P., Ntoumanis, N., Gardner, B., \& Cheval, B. (2020). Evolution of physical activity habits after a context change: The case of COVID-19 lockdown. British Journal of Health Psychology, 26(4), 1135-1154. https://doi.org/10. 1111/bjhp.12524.

McCormack, G., Giles-Corti, B., Lange, A., Smith, T., Martin, K., \& Pikora, T. J. (2004). An update of recent evidence of the relationship between objective and self-report measures of the physical environment and physical activity behaviours. Journal of Science and Medicine in Sport, 7(1), 81-92. https://doi.org/10.1016/S1440-2440(04)80282-2

McDougall, C. W., Brown, C., Thomson, C., Hanley, N., Tully, M. A., Quilliam, R. S., Bartie, P. J., Gibson, L., \& Oliver, D. M. (2020). From one pandemic to another: Emerging lessons from COVID-19 for tackling physical inactivity in cities. Cities \& Health, 0, 1-4. https:// doi.org/10.1080/23748834.2020.1785165

Niedzwiedz, C. L., O'Donnell, C. A., Jani, B. D., et al. (2020). Ethnic and socioeconomic differences in SARS-CoV-2 infection: Prospective cohort study using UK Biobank. medRxiv. 2020.04.22.20075663. https://doi.org/ 10.1101/2020.04.22.20075663

Niedzwiedz, C. L., O'Donnell, C. A., Jani, B. D., Demou, E., Ho, F. K. Celis-Morales, C., Nicholl, B. I., Mair, F. S., Welsh, P., Sattar, N., Pell, J. P., \& Katikireddi, S. V. (2020). Ethnic and socioeconomic differences in SARS-CoV-2 infection: Prospective cohort study using UK Biobank. BMC Medicine, 18(1), 160. https://doi.org/10.1186/s12916020-01640-8

Palan, S., \& Schitter, C. (2018). Prolific.ac-A subject pool for online experiments. Journal of Behavioral and Experimental Finance, 17, 22-27. https:// doi.org/10.1016/j.jbef.2017.12.004
Pierce, M., Hope, H., Ford, T., Hatch, S., Hotopf, M., John, A., Kontopantelis, E., Webb, R., Wessely, S., McManus, S., \& Abel, K. M. (2020). Mental health before and during the COVID-19 pandemic: A longitudinal probability sample survey of the UK population. The Lancet Psychiatry, 7(10), 883-892. https://doi.org/10.1016/S2215-0366(20)30308-4

Prime Minister's statement on coronavirus (COVID-19): 23 March 2020a. GOV UK. https://www.gov.uk/government/speeches/pm-address-to-thenation-on-coronavirus-23-march-2020 (accessed 15 Dec 2020)

Prime Minister's statement on coronavirus (COVID-19): 10 May 2020b. GOV. UK. https://www.gov.uk/government/speeches/pm-address-to-thenation-on-coronavirus-10-may-2020 (accessed 15 Dec 2020)

$\mathrm{R}$ Core Team. R: A language and environment for statistical computing. $\mathrm{R}$ Foundation for Statistical Computing 2019. https://www.R-project.org/

Rhodes, R. E. (2006). The built-in environment: The role of personality and physical activity. Exercise and Sport Sciences Reviews, 34(2), 83-88. https://doi.org/10.1249/00003677-200604000-00008

Rogers, N. T., Waterlow, N. R., Brindle, H., Enria, L., Eggo, R. M., Lees, S., \& Roberts, C. H. (2020). Behavioral change towards reduced intensity physical activity is disproportionately prevalent among adults with serious health issues or self-perception of high risk during the UK COVID-19 lockdown. Frontiers in Public Health, 8, 575091. https://doi.org/10.3389/ fpubh.2020.575091

Teran-Escobar, C., Forestier, C., Ginoux, C., Isoard-Gautheur, S., Sarrazin, P. Clavel, A., \& Chalabaev, A. (2021). Individual, sociodemographic, and environmental factors related to physical activity during the spring 2020 COVID-19 Lockdown. Front Psychol, 12. https://doi.org/10.3389/ fpsyg.2021.643109

Tison, G. H., Avram, R., Kuhar, P., Abreau, S., Marcus, G. M., Pletcher, M. J., \& Olgin, J. E. (2020). Worldwide effect of COVID-19 on physical activity: A descriptive study. Ann Intern Med, 173, 767-770. https://doi.org/10.7326/ M20-2665

Tucker, P., \& Gilliland, J. (2007). The effect of season and weather on physical activity: A systematic review. Public Health, 121(12), 909-922. https://doi. org/10.1016/j.puhe.2007.04.009

Tudor-Locke, C., Bassett, D. R., Swartz, A. M., Strath, S. J., Parr, B. B., Reis, J. P., DuBose, K. D., \& Ainsworth, B. E. (2004). A Preliminary study of one year of pedometer self-monitoring. Annals of Behavioral Medicine, 28(3), 158-162. https://doi.org/10.1207/s15324796abm2803_3

Waldron, S. Measuring subjective wellbeing in the UK. Office for National Statistics 2010. http://www.mas.org.uk/uploads/artlib/measuringsubjective-wellbeing-in-the-uk.pdf (accessed 15 Dec 2020)

Woods, J. A., Hutchinson, N. T., Powers, S. K., Roberts, W. O., Gomez-Cabrera, M. C., Radak, Z., Berkes, I., Boros, A., Boldogh, I., Leeuwenburgh, C., CoelhoJúnior, H. J., Marzetti, E., Cheng, Y., Liu, J., Durstine, J. L., Sun, J., \& Ji, L. L. (2020). The COVID-19 pandemic and physical activity. Sports Medicine and Health Science, 2(2), 55-64. https://doi.org/10.1016/j.smhs.2020.05.006 